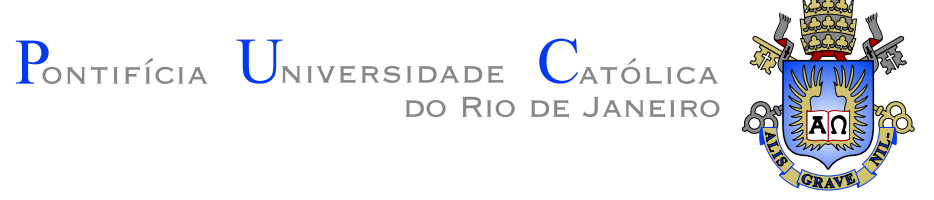

Omar Paranaiba Vilela Neto

Projeto, Otimização, Simulação e Predição de Propriedades de Nanoestruturas através de Técnicas da Inteligência Computacional: Nanotecnologia Computacional Inteligente

Tese de Doutorado

Tese apresentada ao Programa de Pós-graduação em Engenharia Elétrica do Departamento de Engenharia Elétrica da PUC-Rio como requisito parcial para obtenção do título de Doutor em Engenharia Elétrica

Orientador : Prof. Marco Aurélio C. Pacheco Co-Orientador: Prof. André Silva Pimentel 
Omar Paranaiba Vilela Neto

\section{Projeto, Otimização, Simulação e Predição de Propriedades de Nanoestruturas através de Técnicas da Inteligência Computacional: Nanotecnologia Computacional Inteligente}

Tese apresentada como requisito parcial para obtenção do grau de Doutor pelo Programa de Pós-graduação em Engenharia Elétrica do Departamento de Engenharia Elétrica do Centro Técnico Científico da PUC-Rio . Aprovada pela Comissão Examinadora abaixo assinada.

Dr. Marco Aurélio C. Pacheco

Orientador

Departamento de Engenharia Elétrica - PUC-Rio

Dr. André Silva Pimentel

Co-Orientador

Departamento de Química — PUC-Rio

Dr. Mario Norberto Baibich

UFRGS

Dr. Marco Cremona

Departamento de Física - PUC-Rio

Dr. José Roberto M. d'Almeida Departamento de Engenharia de Materiais - PUC-Rio

Dr. Ênio Frota da Silveira Departamento de Física - PUC-Rio

Dr. Pierre M. Esteves UFRJ

Dr. José Eugênio Leal Coordenador Setorial do Centro Técnico Científico PUC-Rio 
Todos os direitos reservados. É proibida a reprodução total ou parcial do trabalho sem autorização da universidade, do autor e do orientador.

\section{Omar Paranaiba Vilela Neto}

Graduou-se em Engenharia de Computação na Pontifícia Universidade Católica do Rio de Janeiro. Mestrado na área de Sistemas de Apoio à Decisão no Departamento de Engenharia Elétrica da Pontifícia Universidade Católica do Rio de Janeiro.

Ficha Catalográfica

Vilela Neto, Omar Paranaiba

Projeto, Otimização, Simulação e Predição de Propriedades de Nanoestruturas através de Técnicas da Inteligência Computacional: Nanotecnologia Computacional Inteligente / Omar Paranaiba Vilela Neto; orientador: Marco Aurélio C. Pacheco; co-orientador: André Silva Pimentel. $-2009$.

$166 \mathrm{f}: ; 30 \mathrm{~cm}$

Tese (Doutorado em Engenharia Elétrica) - Pontifícia Universidade Católica do Rio de Janeiro, Rio de Janeiro, 2009.

Inclui bibliografia.

1. Engenharia Elétrica - Tese.

2. Nanociência;. 3. Nanotecnologia;. 4. Química Computacional;. 5. Inteligência Computacional;. 6. Algoritmos Genéticos;. 7. Redes Neurais Artificiais..

I. Pacheco, Marco Aurélio C.. II. Pimentel, André Silva. III. Pontifícia Universidade Católica do Rio de Janeiro. Departamento de Engenharia Elétrica. IV. Título. 


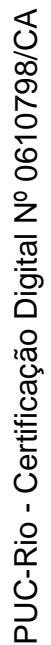

Aos meus pais Paulo Antônio e Cora Alice e à minha esposa Adrissa 


\section{Agradecimentos}

Ao CNPq, FAPERJ e à PUC-Rio pelos auxílios concedidos, sem os quais este trabalho não poderia estar sendo realizado.

Aos meus orientadores Prof. Marco Aurélio C. Pacheco e Prof. André Silva Pimentel pelos ensinamentos e parceria.

Aos professores Ênio da Silveira, Marco Cremona, José Roberto d'Almeida, Patrícia Lustoza e Marley Vellasco pelas discussões, conselhos e ajuda.

Aos meus grandes parceiros neste trabalho Anderson Pires Singulani, Leandro Fontoura Cupertino e Iury Steiner Bezerra pelo trabalho em equipe.

Aos amigos André Vargas, Douglas, Bruno Melo, Rafael Martinez, Renato "Barbosinha", Dilza e Eugênio pelo apoio e incentivo.

Aos amigos do suporte Bernardo, Manuela, André e "Cardosinho" pelos serviços e atenção prestada.

Aos demais amigos do ICA.

Aos funcionários da secretaria e da oficina que ajudaram sempre quando foi necessário.

Ao meu sobrinho João Paulo e à minha irmã Lisa Paula pela torcida, confiança e amor. 


\section{Resumo}

Vilela Neto, Omar Paranaiba; Pacheco, Marco Aurélio C.; Pimentel, André Silva. Projeto, Otimização, Simulação e Predição de Propriedades de Nanoestruturas através de Técnicas da Inteligência Computacional: Nanotecnologia Computacional Inteligente. Rio de Janeiro, 2009. 166p. Tese de Doutorado - Departamento de Engenharia Elétrica, Pontifícia Universidade Católica do Rio de Janeiro.

Esta tese investiga a Nanotecnologia Computacional Inteligente, isto é, o apoio de técnicas de Inteligência Computacional (IC) nos desafios enfrentados pela Nanociência e Nanotecnologia. Por exemplo, utilizam-se as Redes Neurais para construir sistemas de inferência capazes de relacionar um conjunto de parâmetros de entrada com as características finais das nanoestruturas, permitindo aos pesquisadores prever o comportamento de outras nanoestruturas ainda não realizadas experimentalmente. A partir dos sistemas de inferência, Algoritmos Genéticos são então empregados com o intuito de encontrar o conjunto ótimo de parâmetros de entrada para a síntese (projeto) de uma nanoestrutura desejada. Numa outra linha de investigação, os Algoritmos Genéticos são usados para a otimização de parâmetros de funções de base para cálculos ab initio. Neste caso, são otimizados os expoentes das funções gaussianas que compõem as funções de base. Em outra abordagem, os Algoritmos Genéticos são aplicados na otimização de agregados atômicos e moleculares, permitindo aos pesquisadores estudar teoricamente os agregados formados experimentalmente. Por fim, o uso destes algoritmos, aliado ao uso de simuladores, é aplicado na síntese automática de OLEDs e circuitos de Autômatos Celulares com Pontos Quânticos (QCA). Esta pesquisa revelou o potencial da IC em aplicações inovadoras. Os sistemas híbridos de otimização e inferência, por exemplo, concebidos para prever a altura, a densidade e o desvio padrão de pontos quânticos auto-organizáveis, apresentam altos níveis de correlação com os resultados experimentais e baixos erros percentuais (inferior a 10\%). O módulo de elasticidade de nanocompósitos também é previsto por um sistema semelhante e apresenta erros percentuais ainda menores, entorno de 4\%. Os Algoritmos Genéticos, juntamente com o software de modelagem molecular Gaussian03, otimizam os parâmetros de funções que geram expoentes de primitivas gaussianas de funções de base para cálculos hartree-fock, obtendo energias menores do que aquelas apresentadas nas referencias. Em outra aplicação, os Algoritmos Genéticos também se mostram eficientes na busca pelas geometrias de baixa energia dos agregados atômicos de $(\mathrm{LiF}) \mathrm{nLi}+,(\mathrm{LiF}) \mathrm{n}$ e $(\mathrm{LiF}) \mathrm{nF}$-, obtendo uma série de novos isômeros ainda não propostos na literatura. Uma metodologia semelhante é aplicada em um sistema inédito para entender a formação de agregados moleculares de H2O iônicos, partindo-se de agregados neutros. Os resultados mostram como os agregados podem ser obtidos a partir de diferentes perspectivas, formando estruturas ainda não 
investigadas na área científica. Este trabalho também apresenta a síntese automática de circuitos de QCA robustos. Os circuitos obtidos apresentam grau de polarização semelhante àqueles propostos pelos especialistas, mas com uma importante redução na quantidade de células. Por fim, um sistema envolvendo Algoritmos Genéticos e um modelo analítico de OLEDs multicamadas otimizam as concentrações de materiais orgânicos em cada camada com o intuito de obter dispositivos mais eficientes. Os resultados revelam um dispositivo $9,7 \%$ melhor que a solução encontrada na literatura, sendo estes resultados comprovados experimentalmente. Em resumo, os resultados da pesquisa permitem constatar que a inédita integração das técnicas de Inteligência Computacional com Nanotecnologia Computacional, aqui denominada "Nanotecnologia Computacional Inteligente", desponta como uma promissora alternativa para acelerar as pesquisas em Nanociência e o desenvolvimento de aplicações nanotecnológicas.

\section{Palavras-chave}

Nanociência; Nanotecnologia; Química Computacional; Inteligência Computacional; Algoritmos Genéticos; Redes Neurais Artificiais. 


\section{Abstract}

Vilela Neto, Omar Paranaiba; Pacheco, Marco Aurélio C.(Advisor); Pimentel, André Silva. Design, Optimization, Simulation and Prediction of Nanostructures Properties by Computational Intelligence Techniques: Intelligent Computational Nanotechnology. Rio de Janeiro, 2009. 166p. D.Sc. Thesis — Departamento de Engenharia Elétrica, Pontifícia Universidade Católica do Rio de Janeiro.

This thesis investigates the Intelligent Computational Nanotechnology, that is, the support of Computational Intelligence (CI) techniques in the challenges faced by the Nanoscience and Nanotechnology. For example, Neural Networks are used for build Inference systems able to relate a set of input parameters with the final characteristics of the nanostructures, allowing the researchers foresees the behavior of other nanostructures not yet realized experimentally. From the inference systems, Genetic Algorithms are then employees with the intention of find the best set of input parameters for the synthesis (project) of a desired nanostructure. In another line of inquiry, the Genetic Algorithms are used for the base functions optimization used in ab initio calculations. In that case, the exponents of the Gaussian functions that compose the base functions are optimized. In another approach, the Genetic Algorithms are applied in the optimization of molecular and atomic clusters, allowing the researchers to theoretically study the experimentally formed clusters. Finally, the use of these algorithms, use together with simulators, is applied in the automatic synthesis of OLEDs and circuits of Quantum Dots Cellular Automata (QCA). This research revealed the potential of the $\mathrm{CI}$ in innovative applications. The hybrid systems of optimization and inference, for example, conceived to foresee the height, the density and the height deviation of self-assembled quantum dots, present high levels of correlation with the experimental results and low percentage errors (lower to $10 \%$ ). The Young's module of nanocomposites is also predicted by a similar system and presents percentage errors even smaller, around 4\%. The Genetic Algorithms, jointly with the package of molecular modeling Gaussian03, optimize the parameters of functions that generate exponents of primitive Gaussian functions of base sets for hartree-fock calculations, obtaining smaller energies than those presented in the literature. In another application, the Genetic Algorithms are also efficient in the search by the low energy geometries of the atomic clusters of $(\mathrm{LiF}) \mathrm{nLi}+,(\mathrm{LiF})$ $\mathrm{n}$ and $(\mathrm{LiF}) \mathrm{nF}-$, obtaining a set of new isomers yet not propose in the literature. A similar methodology is applied in an unpublished system for understand the formation of molecular cluster of ionic $\mathrm{H} 2 \mathrm{O}$ from neutral clusters. The results show how the clusters can be obtained from different perspectives, forming structures not yet investigate in the scientific area. This work also presents the automatic synthesis of robust QCA circuits. The circuits obtained present high polarization, similar to those proposed by the specialists, but with an important reduction in the quantity of cells. 
Finally, a system involving Genetic Algorithms and an analytic model of multilayer OLEDs optimize the concentrations of organic material in each layer in order to obtain more efficient devices. The results reveal a device $9.7 \%$ better that the solution found in the literature, being these results verified experimentally. In summary, the results of the proposed research allow observe that the unpublished integration of the techniques of Computational Intelligence with Computational Nanotechnology, here named "Intelligent Computational Nanotechnology", emerges as a promising alternative for accelerate the researches in Nanoscince and the development of application in Nanotechnology.

\section{Keywords}

Nanoscience; Nanotechnology; Computational Chemistry; Computational Intelligence; Genetic Algorithm; Artificial Neural Network. 


\section{Sumário}

1 Introdução $\quad \mathbf{1 8}$

1.1 Motivação 18

$\begin{array}{lll}1.2 & \text { Objetivos } & 20\end{array}$

1.3 Contribuições 20

1.4 Descrição do Trabalho 22

1.5 Organização do Trabalho 23

2 Nanotecnologia Computacional $\quad 24$

$\begin{array}{lll}2.1 & \text { Introdução } & 24\end{array}$

2.2 Taxonomia da Nanociência e Nanotecnologia 27

2.3 Modelagem Molecular 28

2.4 Nanoinformática 37

2.5 Simulação de Nanodispositivos $\quad 39$

2.6 Computação de Alto Desempenho 39

2.7 Computação Inspirada na Nanotecnologia 40

3 Técnicas de Inteligência Computacional $4 \mathbf{4 2}$

3.1 Computação Evolucionária 42

$\begin{array}{llr}3.2 & \text { Redes Neurais } & 50\end{array}$

4 Inferência e Otimização de Nanoestruturas $\quad 61$

4.1 Introdução 61

4.2 Pontos Quânticos - PQ 62

$\begin{array}{lll}4.3 & \text { Nanocompósitos } & 70\end{array}$

5 Projeto Automático de Nanodispositivos $\quad \mathbf{8 0}$

5.1 Otimização de OLEDs Multicamadas $\quad 80$

5.2 Otimização de Circuitos de QCA Robustos 87

6 Otimização de Funções de Base 96

6.1 Introdução 96

6.2 Criando uma Função de Base 98

6.3 Método da Coordenada Geradora Hartree-Fock - GCHF 99

6.4 Expansão Polinomial 100

6.5 Detalhes Computacionais 101

6.6 Resultados e Discussões 101

7 Otimização de Agregados Atômicos e Moleculares 110

$\begin{array}{ll}7.1 \text { Introdução } & 110\end{array}$

$\begin{array}{lll}7.2 \text { Histórico } & 111\end{array}$

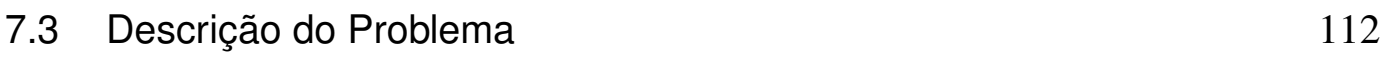

$\begin{array}{lll}7.4 & \text { Agregados de Li e F } & 117\end{array}$

$\begin{array}{ll}7.5 \text { Agregados de } \mathrm{H}_{2} \mathrm{O} & 120\end{array}$

8 Conclusões e Trabalhos Futuros 131 
8.1 Trabalhos Futuros 134

$\begin{array}{lr}\text { Referências Bibliográficas } & 137\end{array}$

A Tabela com os Experimentos dos Pontos Quânticos 151

B Tabela com os Experimentos dos Nanocompósitos $\quad 155$

$\begin{array}{ll}\text { C Resultados para os agregados de Li e F } & 160\end{array}$

D Síntese de OLEDs multicamadas $\quad 164$

$\begin{array}{lll}\text { E Publicações } & 165\end{array}$ 


\section{Lista de figuras}

1.1 Áreas de Atuação e desenvolvimento da Nanotecnologia.

2.1 Relação entre o planeta Terra, uma bola de futebol e um fulereno. 24

2.2 Ilustração de escalas abaixo de 1 metro. Destaque para a escala nanométrica (1nm a 100nm).

2.3 Sub-divisões da Nanociência e Nanotecnologia.

2.4 Algumas das ferramentas usadas no desenvolvimento da Nanociência e Nanotecnologia.

2.5 Taxonomia da Nanotecnologia.

3.1 Procedimento padrão do Algoritmo Genético.

3.2 Modelo co-evolucionário genérico.

3.3 Gráfico com a superposição de 4 pulsos retangulares, cada um com largura 100 e altura 0,0025, representando a distribuição de probabilidade para uma variável

3.4 Forma da superposição de 2 pulsos de distribuição de probabilidade após algumas gerações do algoritmo.

3.5 Representação gráfica de um neurônio artificial.

3.6 Exemplo de uma rede neural não recorrente.

3.7 Exemplo de uma rede neural recorrente com duas entradas e duas saídas.

3.8 Treinamento supervisionado de uma rede neural. (Adaptado de (Reed, 1998))

3.9 Validação cruzada. Ilustração de dois momentos distintos do treinamento: generalização da rede (a) e supertreinamento (b).

3.10 Dependência entre o ponto inicial e o erro final obtido.

4.1 Densidade de estado nos materiais semicondutores. De cima para baixo: material bulk, poço quântico, fio quântico e ponto quântico.

4.2 Diagrama de três formas de crescimento: Frank-van der Merwe (a), Volmer-Weber (b), Stranski-Krastanov (c).

4.3 Imagem de um ponto quântico. Créditos Maurício Pamplona

4.4 Arquitetura da Rede Neural para inferir a altura média dos pontos quânticos.

4.5 Comparação entre as medidas feitas por um AFM e as previsões obtidas pela RNA.

4.6 Variação da altura média dos pontos quânticos com relação ao tempo de deposição e à composição de alumínio.

4.7 Resultado da inferência da densidade de pontos quânticos. De cima para baixo: conjuntos de treinamento, validação e teste.

4.8 Resultado da inferência do desvio da altura dos pontos quânticos. De cima para baixo: conjuntos de treinamento, validação e teste. 
4.9 Procedimento de otimização dos parâmetros para síntese de pontos quânticos.

4.10 Curva da evolução da otimização dos PQ.

4.11 Arquitetura da Rede Neural para inferir o módulo de Young de Nanocompósitos.

4.12 Comparação entre os valores da literatura e as previsões obtidas pela RNA.

4.13 Inferência de um compósito de PMMA/CNF

4.14 Comparação de Modelos para o compósito de argila orgânica (bis(hydroxyl ethyl)-(methyl)) com "nylon-6"

4.15 Comparação de Modelos para o compósito de montmorilonita com SBR

4.16 Comparação de Modelos para o compósito de montemorilonita com NBR

4.17 Comparação de Modelos para o compósito de montemorilonita com CNBR

5.1 a)Representação esquemática de um dispositivo multicamada otimizado. A região graduada é representada em escala de cinza. b) A representação do cromossomo.

5.2 Um exemplo de como criar uma solução para um cromossomo. $\quad 83$

5.3 Eficiência x Densidade de Corrente dos dispositivos 1 e 2

5.4 Uma célula de QCA. As cargas estão localizadas nos pontos pretos.

5.5 Estados de polarização de uma célula de QCA.

5.6 Interferência entre duas células de QCA lado a lado. 88

5.7 Circuito com multicamadas. $\quad 89$

5.8 A arquitetura do modelo de síntese automática. 90

5.9 (A) Circuito sintetizado por especialista; (B) Circuito sintetizado em (VilelaNeto, 2007); (C) Circuito sintetizado neste trabalho.

5.10 (A) Circuito sintetizado por especialista; (B) Circuito sintetizado em (VilelaNeto, 2007); (C) Circuito sintetizado neste trabalho.

7.1 Simplificação da superfície de energia por degraus.

7.2 Exemplo do operador de crossover criado por Deaven e Ho.

7.3 Fluxograma da otimização de agregados atômicos e moleculares. 113

7.4 Cromossomo do agregado iônicos.

7.5 Cromossomo do agregado molecular. 113

$\begin{array}{ll}7.6 & \text { Dois agregados genitores. } \\ 7.7 & 115\end{array}$

$\begin{array}{lll}7.7 & \text { Dois filhos inválidos. } & 116\end{array}$

7.8 Dois novos indivíduos gerados. 116

7.9 Formação de agregados de água positivos através da perda de um elétron.

7.10 Formação de agregados de água iônicos através da agregação de moléculas iônicas.

7.11 O cromossomo para encontrar a posição mais estável do íon na superfície do agregado e a figura ilustrativa do problema em 2D. 


\section{Lista de tabelas}

3.1 Funções de ativação e suas respectivas derivadas. 52

4.1 Valores fixados para gerar a figura 4.6. 67

4.2 Conjunto ótimo de parâmetros encontrado pelo AG. 71

4.3 Normalização dos dados para a RNA.

4.4 Resultado obtido pelo AG para o melhor nanocompósito. 79

5.1 Validação do Modelo de concentração graduada. Os parâmetros n e n' são usadas para calcular a mobilidade na região graduada. O modelo calcula a relação $V / J^{0.5}$.

5.2 Resultados da otimização por AG. Os valores da coluna "anterior" se referem a (Gusso, 2004).

5.3 Comparação entre melhor configuração otimizada por AG, melhor configuração de (Gusso, 2004) e melhor configuração de (Ma, 2002). Em negrito o melhor resultado para cada caso.

6.1 A primeira coluna apresenta o átomo, juntamente com a multiplicidade e termo de simetria. A segunda coluna apresenta o tamanho das funções de base. As últimas três colunas mostram o tamanho do cromossomo para cada experimento.

6.2 Tamanho das sequências aritméticas usadas para gerar os expoentes das funções gaussianas.

6.3 Diferença (micro-hartree) entre as energias obtidas e a energia do estado fundamental dos átomos da primeira linha da tabela periódica. A última coluna mostra o estado fundamental de cada átomo.

6.4 Os cromossomos de todas as otimizações.

105

6.5 O cromossomo da otimização do Ne. Genes variam de 0 a $4 . \quad 106$

6.6 Número de Gerações nos experimentos.

106

6.7 Diferenças de energia (micro-hartree) entre os resultados obtidos pelo polinômio sugerido em (Klobukowski, 1994) e o estado fundamental para os átomos de $\mathrm{C}$ e Ne.

6.8 Comparação dos resultados obtidos pelo polinômio sugerido em (Klobukowski, 1994) com aqueles otimizados neste trabalho. Todos para o átomo de C.

6.9 Comparação dos resultados obtidos pelo polinômio sugerido em (Klobukowski, 1994) com aqueles otimizados neste trabalho. Todos para o átomo de $\mathrm{Ne}$.

6.10 Resultados obtidos para o átomo de neônio. 109

7.1 Aptidão dos indivíduos.

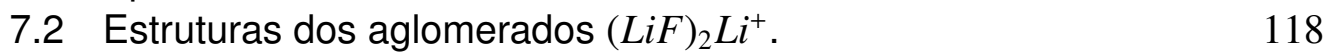

$\begin{array}{lll}7.3 & \text { Estruturas dos aglomerados }(\mathrm{LiF})_{3} \mathrm{Li}^{+} \text {. } & 118\end{array}$

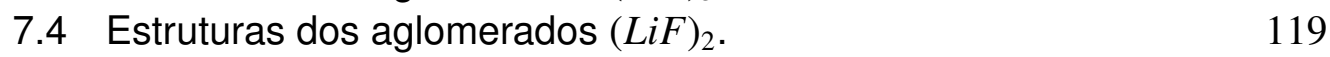

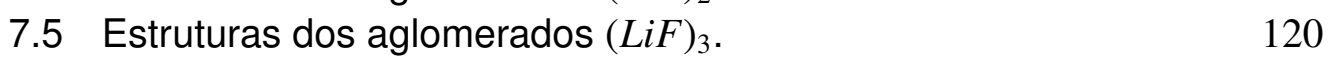




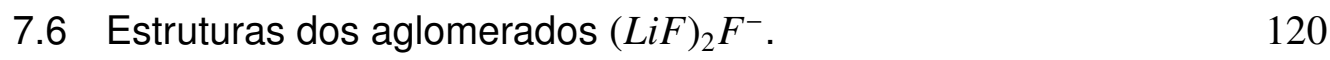

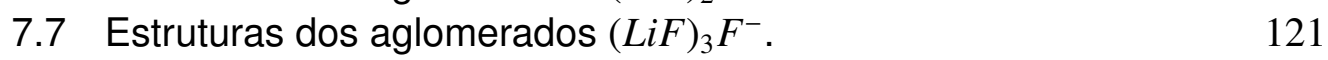

$\begin{array}{lll}7.8 & \text { Estruturas dos aglomerados }\left(\mathrm{H}_{2} \mathrm{O}\right)_{n} . & 123\end{array}$

$\begin{array}{ll}7.9 \text { Estruturas dos aglomerados }\left(\mathrm{H}_{2} \mathrm{O}\right)_{n}^{+} \text {. } & 124\end{array}$

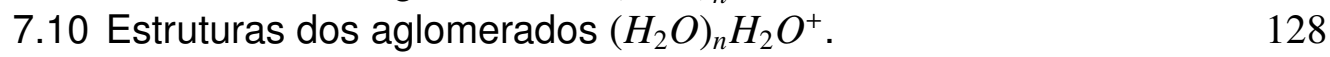

7.11 Estruturas dos aglomerados $\left(\mathrm{H}_{2} \mathrm{O}\right)_{n} \mathrm{H}_{3} \mathrm{O}^{+}$. 129

7.12 Estruturas dos aglomerados $\left(\mathrm{H}_{2} \mathrm{O}\right)_{n} \mathrm{H}_{3} \mathrm{O}^{+}$. 130

B.1 Dados retirados de tabelas disponíveis na literatura. 156

B.2 Dados retirados de tabelas disponíveis na literatura. (continuação) 157

B.3 Dados retirados de gráficos disponíveis na literatura. 158

B.4 Dados retirados de gráficos disponíveis na literatura. (continuação) 159

C.1 Estruturas dos aglomerados $(\mathrm{LiF})_{4} \mathrm{Li}^{+}$. 160

C.2 Estruturas dos aglomerados $(\mathrm{LiF})_{4}$. 161

C.3 Estruturas dos aglomerados $(\mathrm{LiF})_{4} \mathrm{~F}^{-}$. 162

C.4 Estruturas dos aglomerados $(\mathrm{LiF})_{5} F^{-}$. 163 


\section{Siglas}

\begin{tabular}{|c|c|c|}
\hline & AFM & Microscópio de Força Atômica \\
\hline & AEIQ & Algoritmo Evolucionário com Inspiração Quântica \\
\hline & AEIQ-R & Algoritmo Evolucionário com Inspiração Quântica Real \\
\hline & $\mathrm{AG}$ & Algoritmos Genéticos \\
\hline & AGBS & Funções de base gaussianas adaptadas \\
\hline & AGIQ & Algoritmo Genético com Inspiração Quântica \\
\hline & BFGS & Broyden-Fletcher-Goldfarb-Shanno \\
\hline \multirow{23}{*}{ 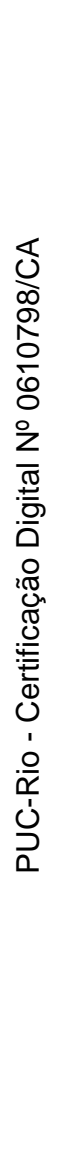 } & CFRP & Fibra de carbono reforçada com polímero \\
\hline & CGTO & Orbitais do tipo gaussiana contraídos \\
\hline & DFP & Davidon-Fletcher-Powell \\
\hline & DFT & Teoria do Funcional da Densidade \\
\hline & $\mathrm{DM}$ & Dinâmica Molecular \\
\hline & DOGBS & Funções de base gaussianas com otimização dupla \\
\hline & EHW & Hardware Evolucionário \\
\hline & ETM & Material transportador de elétron \\
\hline & FPGA & Field Programmable Gate Array \\
\hline & GCHF & Coordenada geradora Hartree-Fock \\
\hline & GPU & Unidade de procesamento gráfico \\
\hline & GTF & Funções de bade do Tipo Gaussiana \\
\hline & GTO & Orbitais do tipo gaussiana \\
\hline & HTM & Material transportador de buraco \\
\hline & $\mathrm{IC}$ & Inteligência Computacional \\
\hline & $\mathrm{KS}-\mathrm{SCF}$ & Campo auto consistente de Kohn-Sham \\
\hline & LM & Levenberg-Marquadt \\
\hline & LCAO & Combinação linear dos orbitais atômicos \\
\hline & LUMO & Lowest Unocuppied Molecular Orbital \\
\hline & MAPE & Erro percentual médio absoluto \\
\hline & MBE & Molecular Beam Epitaxy \\
\hline & MC & Monte Carlo \\
\hline & MCG & Método da Coordenada Geradora \\
\hline
\end{tabular}


MLP Perceptron de múltiplas camadas

MSE Erro quadrático médio

MOCVD Metalorganic Chemical Vapor Deposition

OLED Diodo orgânico emissor de luz

PQ Ponto Quântico

PSO Otimizador por enxame de partículas

QCA Autômatos Celulares com Pontos Quânticos

RB Regularização Bayesiana

RHF Hartree-Fock restrito

ROHF Hartree-Fock abero restrito

RNA Redes Neurais Artificiais

SA Simulated Anneling

SCF Campo auto consistente

SOGBS Funções de base gaussianas com otimização simples

SSE Soma dos erros quadráticos

STF Funções de bade do Tipo Slater

STM Microscópio de Tunelamento

STO Orbitais do tipo Slater

TOGBS Funções de base gaussianas com otimização tripla

UGBS Funções de bases gaussianas universais

XC Troca e correlação

ZPE Energia de ponto zero 